\title{
Editorial: Brain-Metabolic Crossroads in Severe Mental Disorders-Focus on Metabolic Syndrome
}

\author{
Virginio Salvi ${ }^{1 *}$ and Tomas Hajek ${ }^{2}$ \\ ${ }^{1}$ Department of Neuroscience, ASST Fatebenefratelli Sacco, Milan, Italy, ${ }^{2}$ Department of Psychiatry, Dalhousie University, \\ Halifax, NS, Canada
}

Keywords: insulin, metabolic syndrome (MetS), cognition, metabolism, brain

\section{Editorial on the Research Topic}

\section{Brain-Metabolic Crossroads in Severe Mental Disorders-Focus on Metabolic Syndrome}

A large body of evidence shows that patients with severe mental illnesses are more frequently affected by metabolic disorders such as diabetes and the metabolic syndrome, which contribute to cardiovascular morbidity and reduced life expectancy (1). In addition, we are learning more about the effects of metabolic pathways and key metabolic hormones on the functioning of the brain. For example, even though insulin is not necessary for the neuronal uptake and utilization

\section{OPEN ACCESS}

Edited and reviewed by: Stephan Zipfel, University of Tübingen,

Germany

*Correspondence: Virginio Salvi

virginiosalvi@gmail.com

Specialty section:

This article was submitted to

Psychosomatic Medicine, a section of the journal

Frontiers in Psychiatry

Received: 19 May 2019 Accepted: 24 June 2019

Published: 12 July 2019

Citation:

Salvi V and Hajek T (2019) Editorial:

Brain-Metabolic Crossroads in

Severe Mental Disorders - Focus

on Metabolic Syndrome.

Front. Psychiatry 10:492.

doi: 10.3389/fpsyt.2019.00492 of glucose in the brain, it has multiple roles in central nervous system, from acting as a growth factor to directly influencing cognitive functions such as memory formation (2). It is thus not surprising that patients with type 2 diabetes display cognitive impairments, even in the absence of macrovascular complications. They are at an increased risk for accelerated brain aging and dementia. Furthermore, diabetes in major psychiatric disorders is not only associated with worse somatic health, but also with poor psychiatric outcomes, poor response to psychiatric medications, and with greater extent of neurostructural and neurochemical brain alterations $(3,4)$.

Besides insulin, other mediators such as total and HDL cholesterol levels play key roles in the brain and were found to have pathoplastic effects on psychiatric disorders: the former has been repeatedly associated with suicide risk (5), the latter with higher prevalence of negative symptoms in schizophrenia (6). Considering the role of these metabolic pathways on the functioning of the brain, it is not surprising that metabolic syndrome, which is highly prevalent in patients with bipolar disorders and schizophrenia (7) and tends to increase over time (8), contributes to cognitive decline (9) and brain ageing (10) in schizophrenia and even interferes with the efficacy of cognitive remediation therapy (11).

The scope of the present Research Topic, then, was to collect papers investigating the complex interplay between brain/cognitive/psychopathological and metabolic disturbances in severe mental disorders. Several eminent researchers contributed to this collection, which includes seven original research articles and three literature reviews. These studies contribute to our knowledge about the interrelationship between cognitive and metabolic disturbances in severe mental illnesses. They highlight the need to rethink psychiatry as a branch of medicine deeply intertwined with physical health. 
The observation that severe mental illnesses are associated with adverse metabolic profiles draws back to the prepharmacological era. As far back as in late $19^{\text {th }}$ century, the British psychiatrist Henry Maudsley noted that "diabetes is a disease that often shows itself in families where insanity prevails" (12). More than one century later the association is further confirmed, among others, by the work of Toma et al., who reported a more frequent family history of cardiovascular disease in families of subjects with bipolar disorder than in families of healthy controls. Moreover, the authors reported for the first time that the risk of cardiovascular disease increased in families with a family history for bipolar disorder, suggesting common pathogenetic pathways to be investigated in genetic studies.

Besides the common genetic factors, the comorbidity between mental and metabolic disorders may also be explained by common environmental factors. Stress can both influence the expression and severity of mental disorders and contribute to the development of insulin resistance and metabolic syndrome. To address this, Sun et al. conducted a cutting-edge study correlating levels of childhood abuse with glucose metabolism and resting-state functional connectivity in a sample of overweight adolescents with depression. The authors found that high level of abuse was associated with dysfunctional connectivity between amygdala, precuneus, and nucleus accumbens. This connectivity pattern was, in turn, associated with decreased levels of glycemia and insulinemia, possibly reflecting a compensatory response in early age. Interestingly, other studies found association between insulin resistance and early life trauma in depressed individuals (13), so we need more research to clarify the existence and directions of these associations and the factors that may mediate/ moderate them.

In another article by Knytl et al., cortisol and other neurosteroids did not qualify as endophenotypes in schizophrenia: although cognitive functions were impaired in patients versus siblings and controls, stress hormone cortisol and other steroids were neither different between the groups nor found to mediate the association with cognitive function. It should be noted, however, that the high number of comparisons and the small sample size lowered the statistical power. It would still be interesting to investigate the effects of neurosteroids on cognition in larger samples.

Increased stress hormones, pro-inflammatory mediators, and alterations in insulin signaling, seem nevertheless to convey increased risk for both metabolic and mental disorders, as Lyra e Silva and colleagues comprehensively describe in their review. The authors concluded their work by discussing which medications used to treat metabolic disorders also exert positive effects on cognition or psychopathology. To this end, liraglutide, an agonist at GLP-1 receptor used to control type 2 diabetes, is one of the most promising drugs. Liraglutide improved overall cognitive performances in a group of subjects with mood disorders (14), and several trials testing its effect on Alzheimer's dementia are ongoing. In this Research Topic, Cuomo et al. evaluated its efficacy in reducing weight in severely obese patients with depressive and bipolar disorders. In their study, liraglutide was effective in $50 \%$ of the treated patients, who lost an average of $10 \mathrm{~kg}$ during the 6 months of treatment. Since liraglutide is still a very expensive antidiabetic medication, studying whether it also has pro-cognitive or pathoplastic effects might create an even stronger impetus for its use in participants with severe mental disorders.

Aside from these other factors, antipsychotic medications are among the strongest contributors to weight gain and diabetes in subjects with psychiatric illnesses. Some antipsychotics were also thought to contribute to worse cognition in patients with schizophrenia (15), although it is challenging to disentangle the effects of medications from the effects of illness. The review by Mackenzie et al. comprehensively summarizes the interplay between antipsychotic use, metabolic effects, and cognition in schizophrenia. Early in the course of illness, antipsychotics seem to convey benefits to cognition by countering the detrimental effects of mental disorders. However, in the long run, as their effect on metabolism grows, their continuous use may start to negatively affect cognitive functioning.

Attempts to reduce the metabolic impact of antipsychotics are thus warranted. In their retrospective analysis of a cohort of veterans, Chipchura et al. assessed for the first time the impact of time of administration on the metabolic profile of aripiprazole. Interestingly, nighttime administration led to a worse metabolic profile in terms of reduced HDL cholesterol. According to the authors, the effect might be mediated by the blocking of pancreatic D2/D3 receptors, leading to a disinhibition of insulin release during nighttime, eventually reducing lipolysis and altering the lipid profile.

In contrast to antipsychotics, antidepressant medicationshave usually been regarded as neutral in their effects on metabolism. The review by Gramaglia et al. indeed does not find an association between antidepressants and metabolic syndrome, with the notable exception of anti-histaminergic antidepressants such as the tricyclics or mirtazapine, which had previously been associated with abdominal obesity and reduction in HDL levels (16).

The impact of treatment on metabolic markers has been extensively studied. More recently, researches have started to examine how metabolic disorders can affect the psychopathological or cognitive outcomes in major mental disorders. For instance, insulin resistance is associated with resistance to treatment with lithium salts (17). In keeping with this trend, Soontornniyomkij et al. showed that in schizophrenia, insulin resistance was associated with a preponderance of negative symptoms. In a large sample of suicide attempters, Aguglia et al. added further evidence about the association between low total cholesterol blood levels and suicide attempts. For the first time, they also showed that low total cholesterol may be linked with a higher lethality of the suicide attempt. This may perhaps be related to the reduction of serotonin transporters in subjects with low cholesterol levels, eventually resulting in higher levels of impulsive behaviors.

Taken together, the high-quality contributions gathered in this Research Topic covered several aspects of the reciprocal influences between metabolism and cognition/psychopathology 
in severe mental illness, and constitute a step ahead in this intriguing and expanding area of research.

\section{AUTHOR CONTRIBUTIONS}

VS and TH equally contributed to designing and writing the manuscript, and approved it for publication.

\section{REFERENCES}

1. Correll CU, Solmi M, Veronese N, Bortolato B, Rosson S, Santonastaso P, et al. Prevalence, incidence and mortality from cardiovascular disease in patients with pooled and specific severe mental illness: a large-scale metaanalysis of 3,211,768 patients and 113,383,368 controls. World Psychiatry (2017) 16(2):163-80. doi: 10.1002/wps.20420

2. Kullmann S, Heni M, Hallschmid M, Fritsche A, Preissl H, Häring HU. Brain insulin resistance at the crossroads of metabolic and cognitive disorders in humans. Physiol Rev (2016) 96(4):1169-209. doi: 10.1152/physrev. 00032.2015

3. Hajek T, Calkin C, Blagdon R, Slaney C, Alda M. Type 2 diabetes mellitus: a potentially modifiable risk factor for neurochemical brain changes in bipolar disorders. Biol Psychiatry (2015) 77(3):295-303. doi: 10.1016/j. biopsych.2013.11.007

4. Hajek T, McIntyre R, Alda M. Bipolar disorders, type 2 diabetes mellitus, and the brain. Curr Opin Psychiatry (2016) 29(1), 1-6. doi: 10.1097/YCO. 0000000000000215

5. Wu S, Ding Y, Wu F, Xie G, Hou J, Mao P. Serum lipid levels and suicidality: a meta-analysis of 65 epidemiological studies. J Psychiatry Neurosci (2016) 41(1):56-69. doi: 10.1503/jpn.150079

6. Gjerde PB, Dieset I, Simonsen C, Hoseth EZ, Iversen T, Lagerberg TV, et al. Increase in serum HDL level is associated with less negative symptoms after one year of antipsychotic treatment in first-episode psychosis. Schizophr Res (2018) 197:253-60. doi: 10.1016/j.schres.2017.10.042

7. Vancampfort D, Stubbs B, Mitchell AJ, De Hert M, Wampers M, Ward $\mathrm{PB}$, et al. Risk of metabolic syndrome and its components in people with schizophrenia and related psychotic disorders, bipolar disorder and major depressive disorder: a systematic review and meta-analysis. World Psychiatry (2015) 14(3):339-47. doi: 10.1002/wps.20252

8. Salvi V, D’Ambrosio V, Bogetto F, Maina G. Metabolic syndrome in Italian patients with bipolar disorder: a 2-year follow-up study. J Affect Disord (2012) 136(3):599-603. doi: 10.1016/j.jad.2011.10.025

9. Bora E, Akdede BB, Alptekin K. The relationship between cognitive impairment in schizophrenia and metabolic syndrome: a systematic review and meta-analysis. Psychol Med (2017) 47(6):1030-40. doi: 10.1017/ S0033291716003366

\section{FUNDING}

$\mathrm{TH}$ was supported by grants from the Canadian Institutes of Health Research (grant number 142255); the Nova Scotia Health Research Foundation; Brain and Behavioral Research Foundation grant to TH; the Ministry of Health, Czech Republic (grant number 16-32791A).

10. Kolenic M, Franke K, Hlinka J, Matejka M, Capkova J, Pausova Z, et al. Obesity, dyslipidemia and brain age in first-episode psychosis. J Psychiatr Res (2018) 99:151-8. doi: 10.1016/j.jpsychires.2018.02.012

11. Bosia M, Buonocore M, Bechi M, Santarelli L, Spangaro M, Cocchi F, et al. Improving cognition to increase treatment efficacy in schizophrenia: effects of metabolic syndrome on cognitive remediation's outcome. Front Psychiatry (2018) 9:647. doi: 10.3389/fpsyt.2018.00647

12. Maudsley H. The pathology of mind. London: Macmillan (1879).

13. Nasca C, Watson K, Bigio B, Robakis T, Myoraku A, Wroolie T, et al. Childhood trauma and insulin resistance in patients suffering from depressive disorders. Exp Neurol (2019) 315:15-20. doi: 10.1016/j.expneurol.2019.01.005

14. Mansur RB, Ahmed J, Cha DS, Woldeyohannes HO, Subramaniapillai M, Lovshin J, et al. Liraglutide promotes improvements in objective measures of cognitive dysfunction in individuals with mood disorders: a pilot, open-label study. J Affect Disord (2017) 207:114-20. doi: 10.1016/j.jad.2016.09.056

15. Veselinović T, Scharpenberg M, Heinze M, Cordes J, Mühlbauer B, Juckel G, et al. NeSSy Study Group. Disparate effects of first and second generation antipsychotics on cognition in schizophrenia-findings from the randomized NeSSy trial. Eur Neuropsychopharmacol (2019) 29(6):720-39. doi: 10.1016/j.euroneuro.2019.03.014

16. Salvi V, Barone-Adesi F, D’Ambrosio V, Albert U, Maina G. High H1-affinity antidepressants and risk of metabolic syndrome in bipolar disorder. Psychopharmacology (Berl) (2016) 233(1):49-56. doi: 10.1007/s00213-015-4085-9

17. Calkin CV, Ruzickova M, Uher R, Hajek T, Slaney CM, Garnham JS, et al Insulin resistance and outcome in bipolar disorder. Br J Psychiatry (2015) 206(1):52-7. doi: 10.1192/bjp.bp.114.152850

Conflict of Interest Statement: The authors declare that the research was conducted in the absence of any commercial or financial relationships that could be construed as a potential conflict of interest.

Copyright (c) 2019 Salvi and Hajek. This is an open-access article distributed under the terms of the Creative Commons Attribution License (CC BY). The use, distribution or reproduction in other forums is permitted, provided the original author(s) and the copyright owner(s) are credited and that the original publication in this journal is cited, in accordance with accepted academic practice. No use, distribution or reproduction is permitted which does not comply with these terms. 